\title{
Line broadening caused by Coulomb carrier-carrier correlations and dynamics of carrier capture and emission in quantum dots
}

Uskov, Alexander V; Magnúsdóttir, Ingibjörg; Tromborg, Bjarne; Mørk, Jesper; Lang, R.

Published in:

Applied Physics Letters

Link to article, DOI:

$10.1063 / 1.1401778$

Publication date:

2001

Document Version

Publisher's PDF, also known as Version of record

Link back to DTU Orbit

Citation (APA):

Uskov, A. V., Magnúsdóttir, I., Tromborg, B., Mørk, J., \& Lang, R. (2001). Line broadening caused by Coulomb carrier-carrier correlations and dynamics of carrier capture and emission in quantum dots. Applied Physics Letters, 79(11), 1679-1681. https://doi.org/10.1063/1.1401778

\section{General rights}

Copyright and moral rights for the publications made accessible in the public portal are retained by the authors and/or other copyright owners and it is a condition of accessing publications that users recognise and abide by the legal requirements associated with these rights.

- Users may download and print one copy of any publication from the public portal for the purpose of private study or research.

- You may not further distribute the material or use it for any profit-making activity or commercial gain

- You may freely distribute the URL identifying the publication in the public portal 


\title{
Line broadening caused by Coulomb carrier-carrier correlations and dynamics of carrier capture and emission in quantum dots
}

\author{
A. V. Uskovi) \\ Lebedev Physical Institute, Leninsky prospect 53, 117924, Moscow, Russia and Research Center COM, \\ Technical University of Denmark, Building 345v, DK-2800 Lyngby, Denmark \\ I. Magnusdottir, B. Tromborg, and J. Mørk \\ Research Center COM, Technical University of Denmark, Building 345v, DK-2800 Lyngby, Denmark \\ R. Lang \\ Department of Applied Physics, Tokyo University of Agriculture and Technology, Koganei-shi 184-8588, \\ Japan and CREST, Japan Science and Technology Corporation, Toyko, Japan
}

(Received 30 March 2001; accepted for publication 13 July 2001)

\begin{abstract}
Mechanisms of pure dephasing in quantum dots due to Coulomb correlations and the dynamics of carrier capture and emission are suggested, and a phenomenological model for the dephasing is developed. It is shown that, if the rates of these capture and emission processes are sufficiently high, significant homogeneous line broadening of the order of several meV can result. (C) 2001 American Institute of Physics. [DOI: 10.1063/1.1401778]
\end{abstract}

The characteristics of the optical spectra of quantum dots (QDs) and, in particular, the widths of the spectral lines can strongly affect the properties of QD based optoelectronic devices. ${ }^{1}$ At low carrier densities, where the QD has only one electron and one hole in the ground state, the QD luminescence spectrum is a narrow line with a width defined mainly by the interaction of QD carriers with phonons ${ }^{2-4}$ and by temperature fluctuations. ${ }^{5}$ At higher carrier densities, when the quantity of carriers inside the QD becomes larger than one electron-hole pair, and/or carriers appear in layers surrounding the QD [e.g. in the wetting layer (WL)], this ground state single exciton line shifts, and new lines appear in the QD spectrum due to Coulomb carrier-carrier correlations. ${ }^{6}$

Coulomb interactions in QD structures can also lead to broadening of QD spectral lines. In particular, elastic collisions of WL carriers with QD carriers can result in homogeneous line broadening of the order of several meV at very moderate WL carrier densities. ${ }^{7}$ Matsuda et al. observed ${ }^{8}$ that, for increasing carrier density excitation (pumping) of a QD structure, broadening of the ground state exciton line occurs in parallel with an increase of intensity of the excited exciton line. This experimental fact can be considered as an indication that the origin of the additional broadening is Coulomb carrier-carrier correlations inside the QD.

One should note that Coulomb correlations in QDs are usually considered at given numbers of carriers in the QD and neglect the continuous spectrum of allowed states above the confined energy levels. ${ }^{6}$ Naturally, such an approach gives only discrete energy levels, and is unable to result in line broadening due to Coulomb interaction between the carriers. In reality, the QD is an open system, and the number of carriers in the QD changes over time due to carrier capture into the QD from two-dimensional (2D) or three-dimensional (3D) barrier states and by carrier emission from the QD into

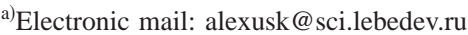

the barrier states. Because of Coulomb correlations inside the QD, the changes in the electron and hole numbers in the QD imply that the transition frequency $\omega$ of the basic exciton line also changes in time, $\omega=\omega(t)$. In fact, this frequency jumps among values corresponding to the ground state single exciton, the charged exciton, the biexciton and so on. Such frequency fluctuations imply pure dephasing of the radiating dipole of the transition considered, and can lead to homogeneous broadening of the QD spectrum, ${ }^{9}$ if these fluctuations are sufficiently fast.

In this letter, we describe two specific mechanisms of homogeneous broadening in a QD due to Coulomb correlations and dynamics of carrier capture and emission in QD structures, and develop a simple model for this broadening. In particular, we show that, if the rates of these capture and emission processes are sufficiently high, then substantial homogeneous QD line broadening (of the order of several $\mathrm{meV}$ ) can take place. We believe that our simple method can be applied to a wider range of similar mechanisms.

Figure 1 illustrates processes that can lead to jumps of the transition frequency $\omega$ of the considered line between $t w o$ values. In Fig. 1(a), one electron and one hole are in their ground states e 1 and $\mathrm{h} 1$ in the QD, respectively. Electron A is captured (for instance, due to electron-phonon interaction) from the barrier to state e 2 in the QD, and is then emitted back into the barrier. When electron $\mathrm{A}$ is in the barrier, its wave function extends over a large volume, so the electron charge density is negligible, and Coulomb effects of electron A on the QD energy levels can be discarded. In this case, the electron-hole pair radiates at the single exciton frequency $\omega_{0}^{\prime}$. If electron $\mathrm{A}$ is at the e2 level, we have a charged exciton configuration with the radiation frequency $\omega_{1}^{\prime}$ of the ground state electron-hole pair, and $\omega_{1}^{\prime} \neq \omega_{0}^{\prime}$ due to Coulomb interaction of the pair with electron A. Thus, because of the carrier capture and emission processes the radiation frequency $\omega$ fluctuates $[\omega=\omega(t)]$ between two values, $\omega_{0}^{\prime}$ and $\omega_{1}^{\prime}$. If $n^{\prime}$ is the population of the e 2 level, the radiation frequency is $\omega=\omega_{0}^{\prime}$ at $n^{\prime}=0$ and $\omega=\omega_{1}^{\prime}$ at $n^{\prime}=1$. 


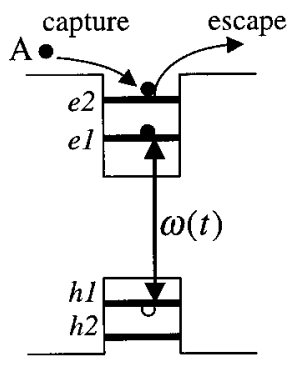

(a)

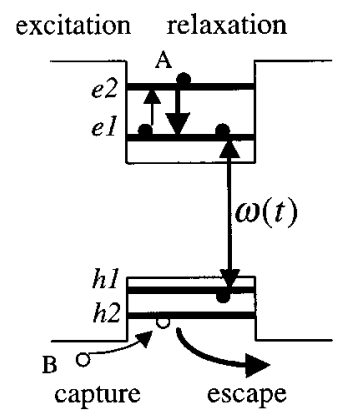

(b)
FIG. 1. Illustration of the mechanisms of line broadening. Two electron (e1 and e2) and hole (h1 and h2) levels are shown in the schematic QD band diagrams. The optical transition e1-h1 with frequency $\omega(t)$ is considered. (a) Electron A is captured into the QD e2 level, and then escapes into the barrier. (b) Hole B is captured into the h2 level with the excitation of electron A from level e1 to level e2, and is subsequently emitted into the barrier with relaxation of electron A from level e2 to level e1.

In Fig. 1(b), one electron and one hole are in their ground states. This pair radiates with frequency $\omega_{0}^{\prime \prime}$ if electron A is in level e1 and hole B is located in a barrier state (the charged exciton configuration). Coulomb interaction of electron A with hole B in the barrier results in hole B being captured into the excited $\mathrm{h} 2$ state and electron A being excited to the e 2 state (the Auger process), and we get the two exciton configuration. This capture-excitation process is shown in Fig. 1(b) by thin arrows. The transition frequency of the considered radiating pair $(\mathrm{e} 1-\mathrm{h} 1)$ in this configuration is $\omega_{1}^{\prime \prime}$, and is different from $\omega_{0}^{\prime \prime}$. Coulomb interaction of hole $\mathrm{B}$ and electron A inside the QD can lead to emission of hole $\mathrm{B}$ from the QD and relaxation of electron A back to level e1 (the processes are shown by bold arrows). We see again that due to the capture-excitation and emission-relaxation processes the radiation frequency $\omega=\omega(t)$ fluctuates between $\omega_{0}^{\prime \prime}$ and $\omega_{1}^{\prime \prime}$. If $n^{\prime \prime}$ is the population of levels e2 and h2, i.e., the population of the QD with excited excitons e2-h2, then the radiation frequency is $\omega=\omega_{0}^{\prime \prime}$ at $n^{\prime \prime}=0$ and $\omega=\omega_{1}^{\prime \prime}$ at $n^{\prime \prime}=1$.

Broadening of the QD spectrum due to the mechanisms in Fig. 1 can be described within the framework of the stochastic equation,

$$
\dot{x}=-\gamma x-i \omega(t) x+f(t),
$$

for the complex amplitude $x(t)$ of the radiating dipole. The transition frequency $\omega=\omega(t)$ randomly fluctuates between $\omega_{0}$ and $\omega_{1}\left[\omega_{0}=\omega_{0}^{\prime}, \omega_{1}=\omega_{1}^{\prime}\right.$ for the mechanism in Fig. 1(a) and $\omega_{0}=\omega_{0}^{\prime \prime}, \omega_{1}=\omega_{1}^{\prime \prime}$ for the mechanism in Fig. 1(b)]. Spontaneous emission leads to decay of the oscillator with the rate $\gamma$, and to noise fluctuations described by the Langevin force $f(t)$. The force is assumed to be delta correlated, i.e., $\left\langle f(t) f^{*}(t+\tau)\right\rangle=C \delta(\tau)$, where the angled brackets \langle\rangle indicate an average over fluctuations. The coefficient $C$ is related to the rate $\gamma$ and the intensity of the spectral line. ${ }^{9}$ The $S(\omega)$ spectrum is expressed as $S(\omega)$ $\propto \int_{-\infty}^{+\infty} d t e^{-i \omega t}\left\langle x(t) x^{*}(t+\tau)\right\rangle$. In the calculation of $S(\omega)$ we follow the method in Ref. 10. It is easy to show that where $\langle\widetilde{g}(z)\rangle$ is the Laplace transform of the average Green function $\langle g(t)\rangle$ for Eq. (1): $\langle\widetilde{g}(z)\rangle=\int_{0}^{+\infty} d t e^{i z t}\langle g(t)\rangle$. Thus, the problem of calculating $S(\omega)$ is reduced to finding $\langle\widetilde{g}(z)\rangle$.

We consider the process $\omega(t)$ as a Markov process with two discrete values or, in different terminology, a KuboAnderson process (KAP) with two values. ${ }^{10}$ It means the following. If $P_{0}(t)$ is the probability that $\omega(t)=\omega_{0}[n=0, n$ is the population $n^{\prime}$ of level e2 for the mechanism in Fig. (a), and $n$ is the population $n^{\prime \prime}$ of excited excitons (e2-h2) for the mechanism in Fig. 1(b)], and if $P_{1}(t)$ is the probability that $\omega(t)=\omega_{1}(n=1)$, then

$$
\dot{P}_{0}(t)=-v_{c} P_{0}+v_{e} P_{1} ; \quad \dot{P}_{1}(t)=+v_{c} P_{0}-v_{e} P_{1},
$$

where $v_{c}=1 / \tau_{c}\left(v_{e}=1 / \tau_{e}\right)$ is the capture (emission) rate for electron A in Fig. 1(a) or the capture-excitation (emissionrelaxation) rate for excitons in Fig. 1(b), and $\tau_{c}\left(\tau_{e}\right)$ is the respective capture (emission) time. Equations (3) have the stationary solution $P_{0}^{s}=1-\bar{n}, P_{1}^{s}=\bar{n}$, where $\bar{n}=v_{c} /\left(v_{c}\right.$ $\left.+v_{e}\right)$ is the average population. One can show that, in this stationary regime, the correlation $\langle\delta \omega(t) \delta \omega(t+\tau)\rangle$ for the deviation $\delta \omega=\omega(t)-\langle\omega\rangle$ is given by $\langle\delta \omega(t) \delta \omega(t+\tau)\rangle$ $=\left\langle\delta \omega^{2}\right\rangle e^{-v|\tau|}$ where

$$
v=v_{e} /(1-\bar{n}) .
$$

The average Green's function for this KAP is ${ }^{10}$

$$
\langle\widetilde{g}(z)\rangle_{\mathrm{KAP}}=\frac{\widetilde{g}_{s \mathrm{KAP}}(z+i v)}{1-v \widetilde{g}_{s \mathrm{KAP}}(z+i v)},
$$

where $\widetilde{g}_{s \mathrm{KAP}}(z)$ is the so-called static Green's function,

$$
\widetilde{g}_{s \mathrm{KAP}}(z)=\frac{1-\bar{n}}{\gamma+i\left(\omega_{0}-z\right)}+\frac{\bar{n}}{\gamma+i\left(\omega_{1}-z\right)} .
$$

Substitution of Eqs. (5) and (6) into Eq. (2) gives the $S(\omega)$ spectrum.

We see that the $S(\omega)$ spectrum depends on the average population $\bar{n}$. If $\bar{n}=0(\bar{n}=1)$ the spectrum is Lorentzian, centered at $\omega=\omega_{0}\left(\omega=\omega_{1}\right)$ with a full width at half maximum (FWHM) equal to $2 \gamma$, i.e., there is no additional line broadening at $\bar{n}=0$ and 1 . For $0<\bar{n}<1$, the $S(\omega)$ spectrum can have a more complicated shape, which depends on $\bar{n}$ and the relation among $\gamma, v$ and $\Delta \omega=\left|\omega_{1}-\omega_{0}\right|$. If $v \ll \gamma$,

$$
S(\omega) \propto \frac{1-\bar{n}}{\gamma+i\left(\omega_{0}-\omega\right)}+\frac{\bar{n}}{\gamma+i\left(\omega_{1}-\omega\right)}+c c .
$$

i.e., the spectrum is the sum of two unbroadened Lorentzians at $\omega=\omega_{0}$ and $\omega=\omega_{1}$ with weighting of $1-\bar{n}$ and $\bar{n}$, respectively. This is the so-called static case, and the condition $v$ $\ll \gamma$ means simply that the frequency $\omega(t)$ does not change its value $\left(\omega_{0}\right.$ or $\left.\omega_{1}\right)$ during radiative decay of the optical dipole, which takes place on a time scale of $\sim 1 / \gamma$.

Consider the more interesting case of $v \gg \gamma$. Figure 2 shows the normalized $S(\omega) / S_{\max }$ spectra for different $\bar{n}$ and $v_{e}$. In all these plots, we assume that $\omega_{0}-\omega_{1}=3 \mathrm{meV}$, which is in accordance with the calculations in Ref. 5, and $\gamma=0.06 \mathrm{meV}$. Figure 2 shows that the shape of the $S(\omega)$ spectrum depends strongly on $\bar{n}$ and on the relation between $v_{e}$ and $\Delta \omega$. At $\bar{n}=0$, we have a line at $\omega_{0}$ with width of $2 \gamma$. If $v_{e}=0.3 \mathrm{meV}$ [Fig. 2(a)] $\left(\tau_{e} \sim 3 \mathrm{ps}, v_{e} \ll \Delta \omega\right)$, an increase of $\bar{n}$ results in broadening of the line at $\omega=\omega_{0}$ and the apDownloaded 26 Feb 2010 to 192.38.67.112. Redistribution subject to AIP license or copyright; see http://apl.aip.org/apl/copyright.jsp 


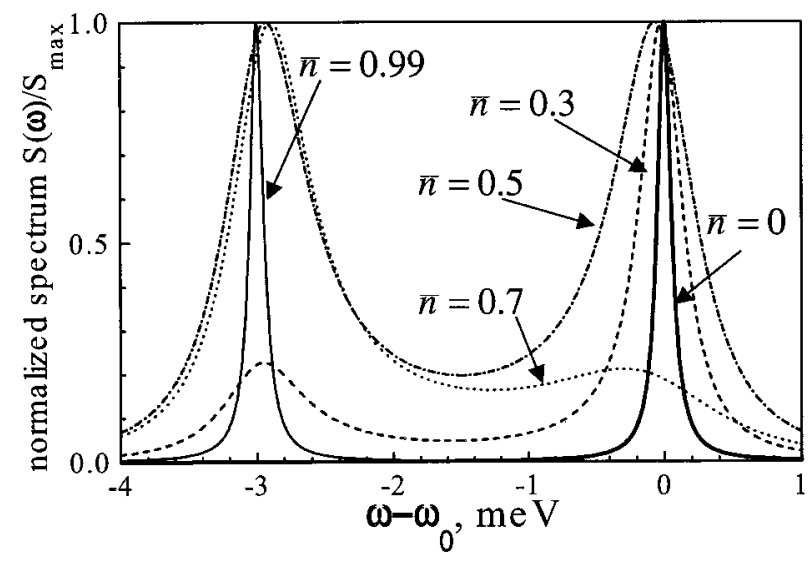

(a)

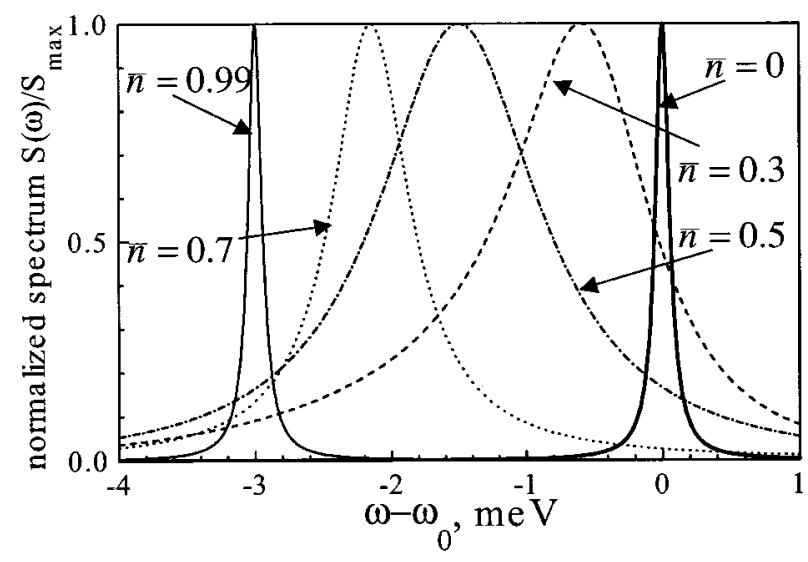

(b)

FIG. 2. Normalized $S(\omega) / S_{\max }$ spectra. In all plots, $\omega_{0}=0, \omega_{1}=-3 \mathrm{meV}$, and $\gamma=0.06 \mathrm{meV}$. Thick solid line: $\bar{n}=0$; dashed line: $\bar{n}=0.3$; dot-dashed line: $\bar{n}=0.5$; dotted line: $\bar{n}=0.7$; thin solid line: $\bar{n}=0.99 . v_{e}=$ (a) 0.3 and (b) $2 \mathrm{meV}$.

pearance of a broad line at $\omega=\omega_{1}$. At $\bar{n}=0.5$, we observe two resolved peaks [dot-dashed line in Fig. 2(a)]. With a further increase of $\bar{n}$, the line at $\omega=\omega_{0}$ disappears, and the line at $\omega=\omega_{1}$ grows and narrows to a width of $2 \gamma$ [thin solid line in Fig. 2(a)]. The case of $v_{e} \ll \Delta \omega$ is thus similar to the static case, Eq. (7), but is accompanied by substantial line broadening of the order of $v_{e}$.

Increasing $v_{e}$ means that the two broadened lines come close to each other, and finally merge. At $v_{e} \geqslant 0.5 \Delta \omega$ $=1.5 \mathrm{meV}$ (corresponding to $\tau_{e} \sim 1 \mathrm{ps}$ ), only one line is observed, and the line shifts from $\omega_{0}$ to $\omega_{1}$ with increasing $\bar{n}$. Figure 2(b) $\left(v_{e}=2 \mathrm{meV}\right)$ illustrates this behavior. When the average population $\bar{n}$ increases from 0 to 0.5 , the line broadens, reaching a maximum width of $\sim 1.5 \mathrm{meV}$ at $\bar{n}=0.5$. A further increase of $\bar{n}$ implies narrowing of the line. One can show that, at $v_{e} \geqslant \Delta \omega$, the line at $\bar{n}=0.5$ is Lorentzian with linewidth of $\sim \Delta \omega^{2} / v$.

Thus, the spectral line shape and its width depend quite strongly on the parameter $v$, which characterizes the population dynamics in the QD, and can be very different from those in the static case [Eq. (7)]. For $v_{e} \geqslant 0.5 \Delta \omega$, one instead of two lines is observed. For the mechanism shown in Fig. 1(a), one can say that the single exciton and the charged exciton merge into one common exciton. Thus, it appears impossible to consider these two excitons separately if the dynamics of carrier capture and emission are fast.

As discussed above, only one line will appear in the QD spectrum, if the emission rate $v_{e}$ is higher than approximately $0.5 \Delta \omega\left(\tau_{e}<2 / \Delta \omega\right)$. In general, the difference frequency $\Delta \omega$ between two excitons depends on the QD size and is of the order of several meV. ${ }^{5}$ It implies that for observation of one merged or composite line, instead of two wellseparated exciton lines, the emission time must be shorter than approximately $1 \mathrm{ps}$. In the mechanism illustrated in Fig. 1(a), such short times may result due to electronlongitudinal optical (LO) phonon interaction in single phonon capture and emission processes. ${ }^{11}$ It implies that the perturbing e2 level must be relatively shallow, with its bound energy less than one LO phonon energy below the continuum band edge. On the other hand, by considering the mechanism in Fig. 1(b), emission times of the order of $\sim 100 \mathrm{fs}$ can be reached, ${ }^{11}$ and the e2 level can be relatively deep (in large QDs). Thus, in large QDs the situation with one broad line instead of two well-resolved lines can be realized with the mechanism of Fig. 1(b), rather than with that of Fig. 1(a). In this case, for $\Delta \omega \sim 10 \mathrm{meV}$ the linewidth will be of the order of $\sim \Delta \omega^{2} / v_{e} \sim 10 \mathrm{meV}$. Note also that the linewidth depends strongly on $\bar{n}$, which for this mechanism is the population of the QD with excited excitons (e2-h2). Since the population also determines the intensity of the luminescence of this excited exciton, broadening of the ground state exciton (e1-h1) occurs concurrently with the increase of the luminescence intensity. This agrees with the observation in Ref. 8 where it was shown that the width of the ground state exciton line starts to increase with pumping from a certain level, and that this additional broadening is correlated with the intensity of the luminescence of the excited exciton line.

In conclusion, the dynamics of carrier capture and emission in quantum dots can significantly modify the optical line shape due to Coulomb interactions and can lead to strong line broadening (of several $\mathrm{meV}$ ).

The work of one of the authors (A.V.U.) was supported by the Russian Federal Program "Integration" (Project No. A0155), by RFBR (Project No. 01-02-17330), by the Danish Research Council within the framework of program "SCOOP," and by the Otto Monsted Foundation.

\footnotetext{
${ }^{1}$ D. Bimberg, M. Grundmann, and N. N. Ledentsov, Quantum Dot Heterostructures (Wiley, New York, 1999).

${ }^{2}$ T. Takagahara, Phys. Rev. B 60, 2638 (1999).

${ }^{3}$ X.-Q. Li and Y. Arakawa, Phys. Rev. B 60, 1915 (1999).

${ }^{4}$ A. V. Uskov, A.-P. Jauho, B. Tromborg, J. Mbrk, and R. Lang, Phys. Rev. Lett. 85, 1516 (2000).

${ }^{5}$ M. Arzberger and M.-C. Amann, Phys. Rev. B 62, 11029 (2000).

${ }^{6}$ See, for instance, U. Hohenester and E. Molinary, Phys. Status Solidi B 201, 19 (2000), and references therein.

${ }^{7}$ A. V. Uskov, K. Nishi, and R. Lang, Appl. Phys. Lett. 74, 3081 (1999).

${ }^{8}$ K. Matsuda, T. Saiki, H. Saito, and K. Nishi, Appl. Phys. Lett. 76, 73 (2000)

${ }^{9}$ M. Sargent, III, M. O. Scaully, and W. E. Lamb, Jr., Laser Physics (Addison-Wesley, New York, 1974).

${ }^{10}$ A. Brissaud and U. Frisch, J. Math. Phys. 15, 524 (1974).

${ }^{11}$ R. Ferreira and G. Bastard, Appl. Phys. Lett. 74, 2818 (1999).
} 